\title{
A novel mutation of COL2A1 in a large Chinese family with avascular necrosis of the femoral head
}

\author{
Zeng Zhang ${ }^{1 \dagger}$, Kechao Zhu ${ }^{1 \dagger}$, Huiyong Dai ${ }^{1}$, Qi Wang ${ }^{1 *}$, Changqing Zhang ${ }^{1}$ and Zhenlin Zhang ${ }^{2}$
}

\begin{abstract}
Avascular necrosis of the femoral head (ANFH) is a debilitating bone disease, characterized by collapse of the femoral head and subsequent loss of hip joint function. Heterozygous mutations in COL2A1 have been identified to cause familial ANFH. Here we report on a large Chinese family with ANFH and a novel heterozygous mutation (c.3517 G > A, p.Gly1173Ser) in exon 50 of COL2A1 in the Gly-X-Y domain. Previously, only five different COL2A1 mutations have been described in patients with familial ANFH. Therefore, our findings provide significant clues to the phenotype-genotype relationships in familial ANFH and may be helpful in clinical diagnosis. Furthermore, these results should assist further studies of the mechanisms underlying collagen diseases.
\end{abstract}

Keywords: COL2A1, Mutation, Type II collagen, Skeletal dysplasia, Cartilage, Avascular necrosis of the femoral head

\section{Introduction}

Type II collagenopathies represent a group of chondrodysplasias which are expressed as a continuous spectrum of phenotypes, ranging from perinatally lethal (Achondrogenesis II; OMIM 200610) to severe (Spondyloepiphyseal dysplasia congenital; OMIM 183900) to those with only mild arthropathy (Stickler dysplasia; OMIM 108300) [1-4]. The common molecular bases of the type II collagenopathies are heterozygous mutations in the type II collagen gene (COL2A1), which encodes the precursor of the type II collagen $\alpha 1$ chain, the most abundant cartilage component [5].

Avascular necrosis of the femoral head (ANFH) is characterized by collapse of the femoral head and subsequent loss of hip joint function. Its clinical manifestations include progressive pain in the groin, pain on exertion,

\footnotetext{
*Correspondence: qiwangsh@163.com

†Zeng Zhang and Kechao Zhu have contributed equally to this work

${ }^{1}$ Department of Orthopedic Surgery, Shanghai Jiao Tong University

Affiliated the Sixth People's Hospital, 600 Yi-Shan Rd., Shanghai 200233,

People's Republic of China

Full list of author information is available at the end of the article
}

a limping gait, and a discrepancy in leg length. Most cases of ANFH are sporadic, and several etiologic factors (including trauma, alcohol, steroids) have been reported to be implicated $[6,7]$. Besides, there are familial cases of ANFH, which may be related to genetic factors. Actually, Liu et al. identified that heterozygous mutations in COL2A1 caused familial ANFH [8]. Thus, familial ANFH belongs to type II collagenopathies and represents the mild end of spectrum.

However, only five different COL2A1 mutations have been described in patients with familial ANFH [9-13]. The genotype-phenotype relationship is still poorly understood. Therefore, the studies of more patients with novel mutations in COL2A1 will be needed for further research to clarify the genotype-phenotype relationship. Here we report one novel mutation in the COL2A1 gene that causes ANFH in a large Chinese family.

\section{Materials and methods Human subjects}

This study was approved by the Ethics Committee of the Shanghai Jiao Tong University Affiliated the Sixth 
People's Hospital. All the participants signed informed consent documents according to the Declaration of Helsinki before entering the study.

A large ANFH pedigree (Fig. 1) with a total of 19 subjects was recruited in the present study. Their clinical and genetic information is listed in Table 1. Family members were examined by 2 independent orthopedic surgeons, and imaging results were reviewed by 2 independent radiologists. ANFH were diagnosed using internationally recognized criteria. Age at onset is defined as age at the first appearance of persistent or recurrent limping and/or groin pain, not explained by another cause. The Ficat classification is used to stage avascular necrosis of the femoral head according to plain radiographs, MRI, and clinical features [14]. The proband (IV9), a 51-yearold woman diagnosed with ANFH and premature hip osteoarthritis, visited our hospital due to groin pain and restricted motion of the both hip joints, which started when the patient was 25 years old. A comprehensive survey was conducted to obtain detailed information of the patient's medical history, physical examination and laboratory examination. The X-ray and MRI revealed that collapsed femoral heads with cystic degeneration and premature hip osteoarthritis in both hips, indicating a Ficat stage IV lesion (Fig. 2A, B). The patient was treated with total hip arthroplasty (Fig. 2C). The X-ray of the patient's spine (Fig. 2D, E) was normal and facial features were unremarkable. There were no obvious abnormalities in the patient's neurological system or limbs.

A total of 10 family members were clinically diagnosed with ANFH and premature hip osteoarthritis. Another two affected family members (IV3 and IV7) also received

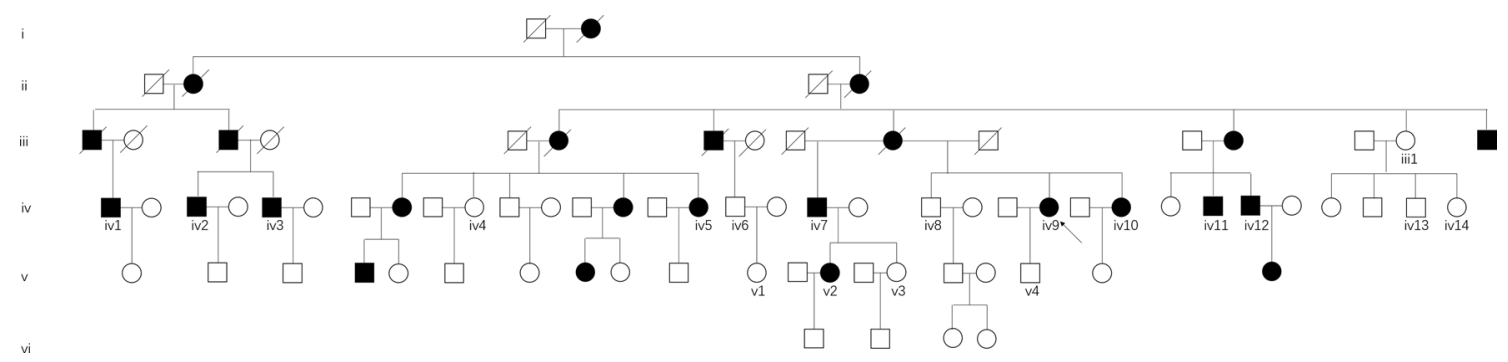

Fig. 1 Pedigree of the family. Black symbols indicate affected individuals, and open symbols unaffected individuals

Table 1 Characteristics of the family members

\begin{tabular}{|c|c|c|c|c|c|c|c|}
\hline & Sex & Age & Disease condition & Ficat classificaion & Age of onset & Height (cm) & Genotype \\
\hline III1 & $\mathrm{F}$ & 73 & Not affected & - & - & 166 & $\mathrm{G} / \mathrm{G}$ \\
\hline IV1 & M & 49 & Affected & IV & 36 & 161 & $\mathrm{G} / \mathrm{A}$ \\
\hline IV2 & M & 51 & Affected & IV & 30 & 160 & $\mathrm{G} / \mathrm{A}$ \\
\hline IV3 & M & 48 & Affected & IV & 25 & 163 & $\mathrm{G} / \mathrm{A}$ \\
\hline IV4 & $\mathrm{F}$ & 58 & Not affected & - & - & 156 & $\mathrm{G} / \mathrm{G}$ \\
\hline IV5 & $\mathrm{F}$ & 52 & Affected & IV & 40 & 155 & $\mathrm{G} / \mathrm{A}$ \\
\hline IV6 & M & 49 & Not affected & - & - & 180 & $\mathrm{G} / \mathrm{G}$ \\
\hline IV7 & M & 62 & Affected & IV & 40 & 163 & $\mathrm{G} / \mathrm{A}$ \\
\hline IV8 & M & 53 & Not affected & - & - & 165 & $\mathrm{G} / \mathrm{G}$ \\
\hline IV9 & $\mathrm{F}$ & 51 & Affected & IV & 38 & 157 & $\mathrm{G} / \mathrm{A}$ \\
\hline IV10 & $\mathrm{F}$ & 49 & Affected & IV & 20 & 155 & $\mathrm{G} / \mathrm{A}$ \\
\hline IV11 & M & 52 & Affected & IV & 40 & 158 & $\mathrm{G} / \mathrm{A}$ \\
\hline IV12 & M & 50 & Affected & IV & 19 & 160 & $\mathrm{G} / \mathrm{A}$ \\
\hline IV13 & M & 50 & Not affected & - & - & 172 & $\mathrm{G} / \mathrm{G}$ \\
\hline IV14 & $F$ & 48 & Not affected & - & - & 165 & $\mathrm{G} / \mathrm{G}$ \\
\hline V1 & $F$ & 25 & Not affected & - & - & 167 & $\mathrm{G} / \mathrm{G}$ \\
\hline V2 & $F$ & 35 & Affected & $\|$ & 26 & 156 & $\mathrm{G} / \mathrm{A}$ \\
\hline V3 & F & 34 & Not affected & - & - & 159 & $\mathrm{G} / \mathrm{G}$ \\
\hline V4 & M & 27 & Not affected & - & - & 165 & $\mathrm{G} / \mathrm{G}$ \\
\hline
\end{tabular}




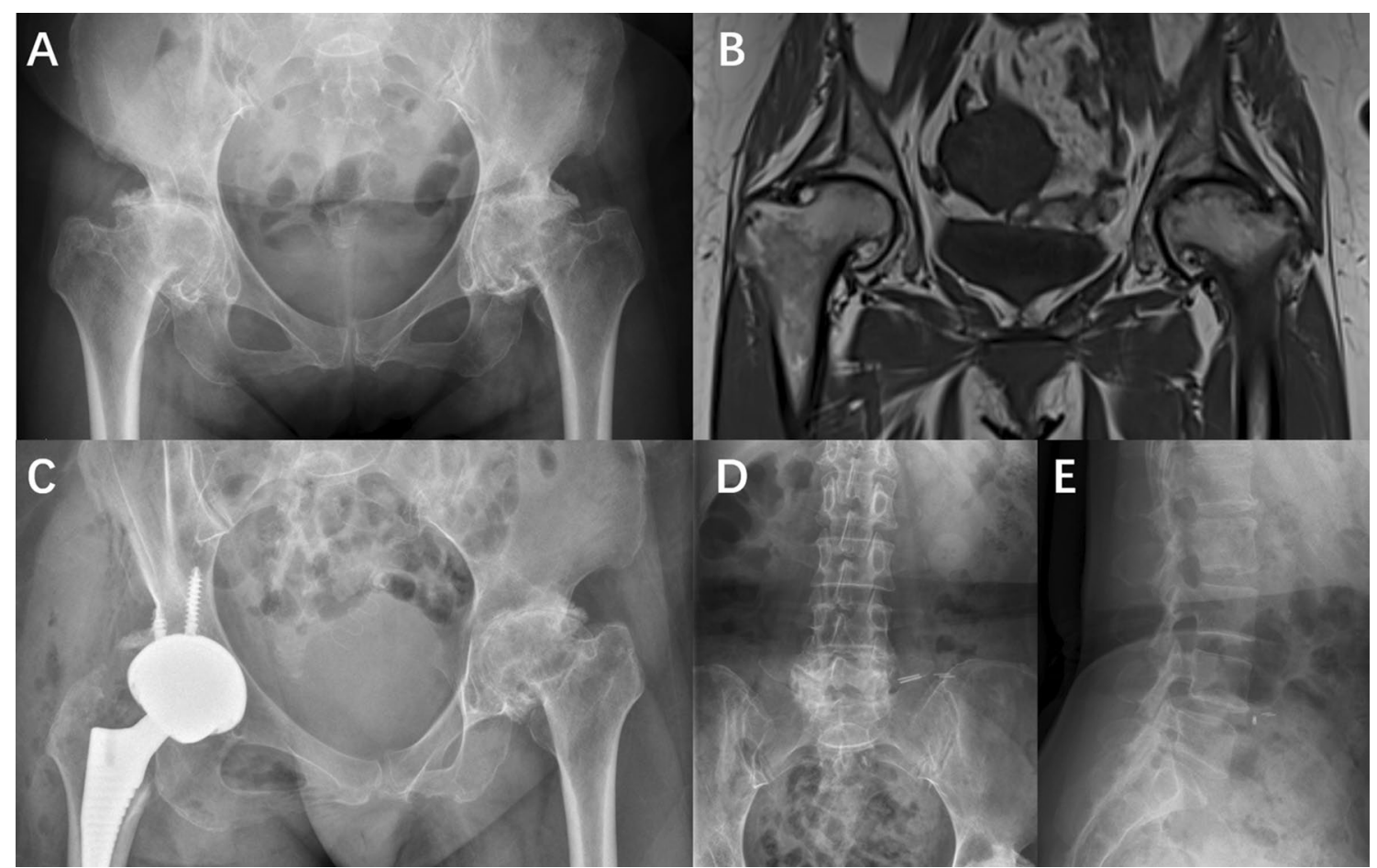

Fig. 2 A, B The X-ray and MRI revealed that collapsed femoral heads with cystic degeneration, and premature hip osteoarthritis in both hips of the proband (IV9). C Post-operation radiograph. D, E The X-ray of the patient's spine indicated normal vertebrae

total hip arthroplasty in our hospital, and the pre-operation and post-operation radiographs were shown in Fig. 3A-D. The pre-operation radiographs also revealed that collapsed femoral heads with cystic degeneration and premature hip osteoarthritis in both hips, which was similar to the proband. It is important to note a relatively young affected family member (V2). This 35-yearold female patient had suffered from groin pain and restricted motion of the left hip joint 10 years ago. Her $\mathrm{X}$-ray (Fig. 3E) showed the surface of the femoral head is smooth with no joint space narrowing, but there is localized increasing of bone density and sclerosis at weightbearing region of the left femoral head. Her MRI (Fig. 3F) showed the crescent-shaped hyperintensity region on $\mathrm{T} 2 \mathrm{~W}$ and lower and uneven signal of necrosis region on T1W in the weight-bearing region of the left femoral head, indicating a Ficat stage II lesion.

\section{Mutation analysis}

Informed consent was obtained from the family and from 250 healthy volunteers before blood sampling and DNA analysis. The DNA was extracted from peripheral white blood cells using conventional methods. The DNA sequence for the COL2A1 gene was obtained from the available online database (GenBank accession No. NC_000012). Primers of the COL2A1 gene were designed using the Primer 3 software (http://frodo.wi.mit.edu/ cgi-bin/primer3/primer3_www.cgi). All exons and their exon-intron boundaries in the COL2A1 gene were amplified via polymerase chain reaction (PCR). Direct sequencing was performed using the BigDye Terminator Cycle Sequencing Ready Reaction Kit, version 3.1 (Applied Biosystems, Foster, CA, USA), and the sequencing was analyzed with an ABI Prism 3130 automated sequencer.

The interpretation of variants was performed according to the American College of Medical genetics and Genomics (ACMG) guideline [15]. The variants were filtered through the following procedures: (1) the allele frequency of variants is required to be less than $1 \%$ or absent from 1000 Genomes Project (The 1000 Genomes Project Consortium 2015). (2) Variants were filtered out when they were synonymous mutation or located in introns without influence on splicing and biological function. PolyPhen-2 (http://genetics.bwh.harvard.edu/pph2/) was used to assess the damaging effects of missense mutations in silico [16].

\section{Statistical analysis}

Continuous variables are presented as means $( \pm S D)$. A Student's $t$ test was used to compare the mean 


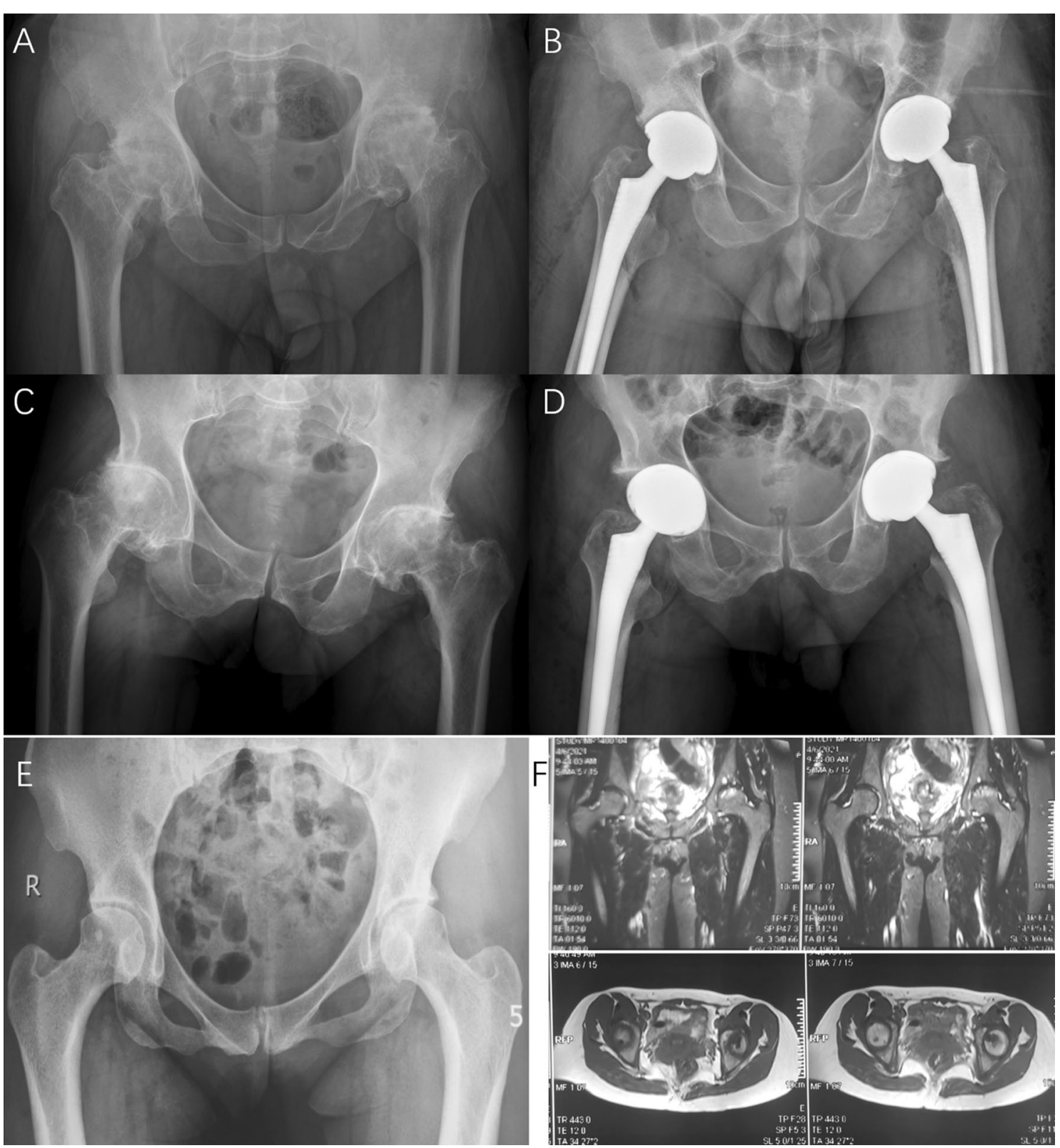

Fig. 3 A, B the pre-operation and post-operation radiographs of IV3. C, D the pre-operation and post-operation radiographs of IV7. The pre-operation radiographs of both patients (IV3 and IV7) showed severe collapse and deformity of the femoral heads with hip joint space narrowing and osteoarthritis. EThe radiograph of $\mathrm{V} 2$ showed the surface of the femoral head is smooth with no joint space narrowing, but there is localized increasing of bone density and sclerosis at weight-bearing region of the left femoral head. $\mathbf{F}$ MRI of V2 showed the crescent-shaped hyperintensity region on T2W and lower and uneven signal of necrosis region on T1W in the left femoral head

values of continuous variables. $p<0.05$ was considered as significant.

\section{Results}

We recruited a Chinese ANFH family with 10 affected members and 9 unaffected members (Fig. 1 and Table 1). The onset age of the disease in the affected members was $31.4 \pm 8.4$ years of old. The height of affected male members was $160.8 \pm 1.9 \mathrm{~cm}$, while it was $170.5 \pm 7.1 \mathrm{~cm}$ in unaffected male members $(p=0.01)$. The height of affected female members was $155.8 \pm 1.0 \mathrm{~cm}$, while it was $162.3 \pm 6.3 \mathrm{~cm}$ in unaffected female members $(p=0.03)$. Therefore, both male 
and female patients in this family have significantly shorter stature than unaffected members.

We screened for the COL2A1 mutation in the proband using PCR followed by direct sequence analysis. All pathogenic and likely pathogenic variants were manually reviewed according to ACMG guidelines. As a result, we identified a heterozygous 1 bp missense (c.3517G > A) in exon 50, which resulted in p.Gly1173Ser (Fig. 4A). Exon and nucleotide numbering was based on RefSeq NM_001844.4, starting at the ATG translation initiation codon. Sanger sequencing was performed in all available family members ( 10 affected members and 9 unaffected members) and 250 healthy volunteers. All affected family members carried the heterozygous mutation. It was not present in the unaffected family members, or in 250 healthy volunteers. These results indicated that this mutation was co-segregated in our family and provides strong evidence for the pathogenicity of this mutation. This mutation is non-conservative, affects evolutionarily highly conserved amino acids from fish to mammals (Fig. 4B). It is predicted to be probably damaging with a score of 0.999 by PolyPhen- 2 in silico analysis.

\section{Discussion}

The present study identified a novel heterozygous c.3517G > A mutation (p.Gly1173Ser) in the COL2A1 gene in a large Chinese family. The main clinical characteristics of the affected patients include ANFH and premature hip osteoarthritis, which has been described by the previous studies $[10,11]$. In recent decades, $>200$ mutations have been identified in the COL2A1 gene, including single substitution, splice-site mutations, insertions and deletions [1]. COL2A1 mutations have been associated with various human disorders, which are collectively termed type II collagenopathies $[17,18]$.

Until now, only six different COL2A1 mutations have been identified in patients with ANFH. As Table 2 shows, five of the six mutations are glycine to serine substitutions in the Gly-X-Y triple-helix, and Gly1170Ser is the hot spot, which has been identified in four families with ANFH.

The nature of the mutations and their localizations in the protein seem to explain the phenotypic differences, at least to a certain extent [18]. Truncating mutations leading to reduced amounts of normal type II collagen are related with mild phenotypes. In contrast, missense mutations, which replace one Gly residue in the Gly-X$\mathrm{Y}$ repeating pattern, are usually related with severe phenotypes. The Gly-X-Y triple-helix motif is crucial for the proper crosslinking of the pro- $\alpha 1$ peptide chain to form functional type II collagen. Mortier et al. reported that there are numerous excessive post-translational modifications in type II collagen in patients carrying a Gly-substituted mutation [20].

The exception is glycine to serine substitutions. Glycine to serine substitutions, unlike glycine to nonserine residue substitutions, produced variable phenotypes, with both inter- and intra-familial phenotypic variation [21, 22]. In type I collagenopathies, the severity of the disease has been correlated with the size and charge of the substituted amino acid, specifically Ala $<$ Ser $<$ Cys $<$ Arg $<$ Glu $<$ Asp $<$ Val, in order from least to

A

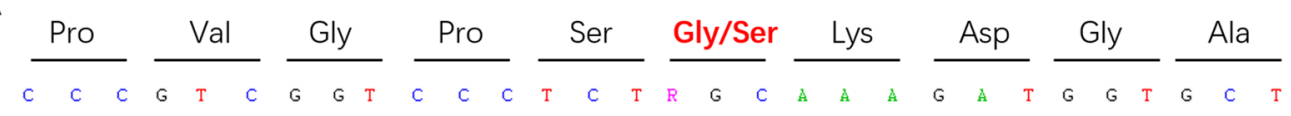

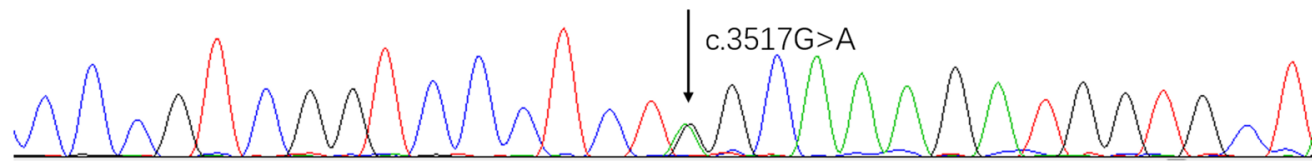

B

Homo sapiens

Mus musculus

Rattus norvegicus

Bos taurus

Xenopus tropicalis

Xenopus laevi

p.Gly1173

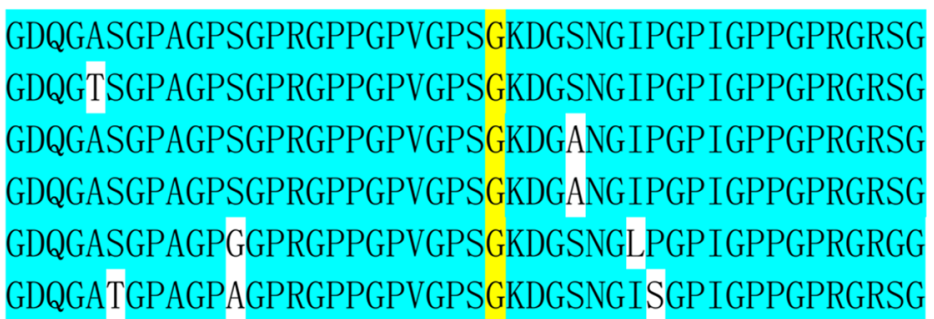

Fig. 4 A The sequencing revealed a heterozygous 1 bp missense (c.3517G > A) in exon 50, which resulted in p.Gly1173Ser. B It is non-conservative, affects evolutionarily highly conserved amino acids from fish to mammals 
Table 2 COL2A1 mutations have been identified in patients with familial ANFH

\begin{tabular}{|c|c|c|c|c|}
\hline Protein & cDNA & Region & Race & References \\
\hline p.Gly582Ser & c. $1744 \mathrm{G}>\mathrm{A}$ & Gly-X-Y & Japanese & Kishiya et al. [19] \\
\hline p.Gly630Ser & c. $1888 \mathrm{G}>\mathrm{A}$ & Gly-X-Y & Chinese & Li et al. [10] \\
\hline p.Gly717Ser & $c .2149 \mathrm{G}>\mathrm{A}$ & Gly-X-Y & Chinese & Liu et al. [8] \\
\hline p.Gly1170Ser & c.3508G > A & Gly-X-Y & Chinese & $\begin{array}{l}\text { Liu et al. [8, 9], Su et al. } \\
\text { [11] and Wang et al. } \\
\text { [13] }\end{array}$ \\
\hline p.Gly1173Ser & c. $3517 \mathrm{G}>\mathrm{A}$ & Gly-X-Y & Chinese & The present study \\
\hline p.Thr1383Met & $c .4148 \mathrm{G}>\mathrm{A}$ & C-propeptide & Unknown & Kannu et al. [12] \\
\hline
\end{tabular}

The variants shown are described using the NM_001844.4 transcript reference sequence

Bold indicates COL2A1 mutation found in this study

most disruptive [23]. The same domain-specific effect may exist in type II collagenopathies. Sobetzko et al. identifiedc.3517G > C mutation leading to Gly1173Arg in COL2A1 in a boy affected with a severe form of spondyloepiphyseal dysplasia [24]. The mutation position is exactly the same with the present study. However, glycine to arginine substitutions usually causes severe phenotypes.

Although most mutations associated with ANFH are glycine to serine substitutions in the Gly-X$\mathrm{Y}$ triple-helix, there is one exception: c.4148G $>\mathrm{A}$ (p.Thr1383Met) in the C-propeptide of COL2A1 gene [12]. C-propeptide mutations typically cause spondyloperipheral dysplasia, characterized by vertebral body abnormalities, hip dysplasia and brachydactyly type E. Therefore, more cases need to be described and more mutations needs to be identified, to clarify the genotype-phenotype relationship.

In summary, we identified a novel heterozygous c.3517G > A mutation (p.Gly1173Ser) in the Gly-X-Y triple-helix motif of COL2A1 in a large Chinese family with ANFH. Our findings will provide clues to the phenotype-genotype relations and may assist not only in the clinical diagnosis of familial ANFH but also in the interpretation of genetic information used for prenatal diagnosis and genetic counseling.

\section{Abbreviations \\ ANFH: Avascular necrosis of the femoral head; COL2A1: Collagen type II alpha 1 chain; SEDC: Spondyloepiphyseal dysplasia congenital.}

\section{Acknowledgements}

Not applicable.

\section{Authors' contributions}

QW designed the study. ZZ and KZ contributed equally to this study. ZZ analyzed the data and wrote the main manuscript text. KZ prepared Figs. 1, 2, 3 and 4. KZ, HD, CZ, ZZ and QW helped revise the manuscript. All the authors read and approved the final manuscript.

\section{Funding}

The study was supported by the National Natural Science Foundation of China (81401764)

\section{Availability of data and materials}

The datasets generated during the current study are available in the Mendeley repository, https://doi.org/10.17632/6j2p5t4xns.1.

\section{Declarations}

Ethics approval and consent to participate

This study was approved by the Ethics Committee of the Shanghai Jiao Tong University Affiliated Sixth People's Hospital. All the participants signed informed consent documents according to the Declaration of Helsinki before entering the study.

\section{Consent for publication}

Written informed consent for publication of clinical details and/or clinical images was obtained from the all the participants.

\section{Competing interests}

The authors declare that they have no competing interests.

\section{Author details}

${ }^{1}$ Department of Orthopedic Surgery, Shanghai Jiao Tong University Affiliated the Sixth People's Hospital, 600 Yi-Shan Rd., Shanghai 200233, People's Republic of China. ${ }^{2}$ Shanghai Clinical Research Center of Bone Disease, Department of Osteoporosis and Bone Diseases, Shanghai Jiao Tong University Affiliated the Sixth People's Hospital, 600 Yi-Shan Rd., Shanghai 200233, People's Republic of China.

Received: 18 February 2021 Accepted: 27 May 2021

Published online: 04 June 2021

\section{References}

1. Barat-Houari M, Sarrabay G, Gatinois V, Fabre A, Dumont B, Genevieve D, Touitou I. Mutation update for COL2A1 gene variants associated with type II collagenopathies. Hum Mutat. 2016;37(1):7-15.

2. Spranger J, Winterpacht A, Zabel B. The type II collagenopathies: a spectrum of chondrodysplasias. Eur J Pediatr. 1994;153(2):56-65.

3. Zhang Z, Zhao SC, He JW, Fu WZ, Zhang CQ, Zhang ZL. Identification of one novel mutation in the C-propeptide of COL2A1 in a Chinese family with spondyloperipheral dysplasia. Gene. 2013;522(1):107-10.

4. Zhang Z, He JW, Fu WZ, Zhang CQ, Zhang ZL. Identification of three novel mutations in the COL2A1 gene in four unrelated Chinese families with spondyloepiphyseal dysplasia congenita. Biochem Biophys Res Commun. 2011:413(4):504-8. 
5. Takahashi E, Hori T, O'Connell P, Leppert M, White R. R-banding and nonisotopic in situ hybridization: precise localization of the human type II collagen gene (COL2A1). Hum Genet. 1990;86(1):14-6.

6. Guerado E, Caso E. The physiopathology of avascular necrosis of the femoral head: an update. Injury. 2016;47(Suppl 6):S16-26.

7. Cohen-Rosenblum A, Cui Q. Osteonecrosis of the femoral head. Orthop Clin North Am. 2019;50(2):139-49.

8. Liu YF, Chen WM, Lin YF, Yang RC, Lin MW, Li LH, Chang YH, Jou YS, Lin PY, Su JS, et al. Type II collagen gene variants and inherited osteonecrosis of the femoral head. N Engl J Med. 2005;352(22):2294-301.

9. Liu F, Xiong Z, Liu Q, Hu J, Li W, Zhang N. COL2A1 mutation (c.3508G > A) leads to avascular necrosis of the femoral head in a Chinese family: a case report. Mol Med Rep. 2018;18(1):254-60.

10. Li N, Yu J, Cao X, Wu QY, Li WW, Li TF, Zhang C, Cui YX, Li XJ, Yin ZM, et al. A novel p.Gly630Ser mutation of COL2A1 in a Chinese family with presentations of Legg-Calve-Perthes disease or avascular necrosis of the femoral head. PLOS ONE. 2014;9(6):e100505.

11. Su P, Li R, Liu S, Zhou Y, Wang X, Patil N, Mow CS, Mason JC, Huang D, Wang Y. Age at onset-dependent presentations of premature hip osteoarthritis, avascular necrosis of the femoral head, or Legg-Calve-Perthes disease in a single family, consequent upon a p.Gly1170Ser mutation of COL2A1. Arthritis Rheum. 2008;58(6):1701-6.

12. Kannu P, O'Rielly DD, Hyland JC, Kokko LA. Avascular necrosis of the femoral head due to a novel C propeptide mutation in COL2A1. Am J Med Genet A. 2011;155A(7):1759-62.

13. Wang $L$, Pan $H$, Zhu ZA. A genetic pedigree analysis to identify gene mutations involved in femoral head necrosis. Mol Med Rep. 2014;10(4):1835-8.

14. Ficat RP. Idiopathic bone necrosis of the femoral head. Early diagnosis and treatment. J Bone Jt Surg Br. 1985;67(1):3-9.

15. Richards S, Aziz N, Bale S, Bick D, Das S, Gastier-Foster J, Grody WW, Hegde $M$, Lyon E, Spector E, et al. Standards and guidelines for the interpretation of sequence variants: a joint consensus recommendation of the American College of Medical Genetics and Genomics and the Association for Molecular Pathology. Genet Med. 2015;17(5):405-24.
16. Adzhubei IA, Schmidt S, Peshkin L, Ramensky VE, Gerasimova A, Bork P, Kondrashov AS, Sunyaev SR. A method and server for predicting damaging missense mutations. Nat Methods. 2010;7(4):248-9.

17. Deng H, Huang X, Yuan L. Molecular genetics of the COL2A1-related disorders. Mutat Res Rev Mutat Res. 2016;768:1-13.

18. Zhang B, Zhang Y, Wu N, Li J, Liu H, Wang J. Integrated analysis of COL2A1 variant data and classification of type II collagenopathies. Clin Genet. 2020;97(3):383-95.

19. Kishiya M, Nakamura Y, Ohishi H, Furukawa K, Ishibashi Y. Identification of a novel COL2A1 mutation (c.1744G>A) in a Japanese family: a case report. J Med Case Rep 2014;8:276.

20. Mortier GR, Weis M, Nuytinck L, King LM, Wilkin DJ, De Paepe A, Lachman RS, Rimoin DL, Eyre DR, Cohn DH. Report of five novel and one recurrent COL2A1 mutations with analysis of genotype-phenotype correlation in patients with a lethal type II collagen disorder. J Med Genet. 2000;37(4):263-71.

21. Nishimura G, Haga N, Kitoh H, Tanaka Y, Sonoda T, Kitamura M, Shirahama

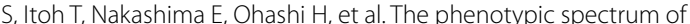
COL2A1 mutations. Hum Mutat. 2005;26(1):36-43.

22. Chen J, Ma X, Zhou Y, Li G, Guo O. Recurrent c.G1636A (p.G546S) mutation of COL2A1 in a Chinese family with skeletal dysplasia and different metaphyseal changes: a case report. BMC Pediatr. 2017;17(1):175.

23. Clements KA, Acevedo-Jake AM, Walker DR, Hartgerink JD. Glycine substitutions in collagen heterotrimers alter triple helical assembly. Biomacromol. 2017;18(2):617-24.

24. Sobetzko D, Eich G, Kalff-Suske M, Grzeschik KH, Superti-Furga A. Boy with syndactylies, macrocephaly, and severe skeletal dysplasia: not a new syndrome, but two dominant mutations (GLI3 E543X and COL2A1 G973R) in the same individual. Am J Med Genet. 2000;90(3):239-42.

\section{Publisher's Note}

Springer Nature remains neutral with regard to jurisdictional claims in published maps and institutional affiliations.
Ready to submit your research? Choose BMC and benefit from:

- fast, convenient online submission

- thorough peer review by experienced researchers in your field

- rapid publication on acceptance

- support for research data, including large and complex data types

- gold Open Access which fosters wider collaboration and increased citations

- maximum visibility for your research: over 100M website views per year

At $B M C$, research is always in progress.

Learn more biomedcentral.com/submissions 